\title{
Multi-objective short-term scheduling of active distribution networks for benefit maximization of DisCos and DG owners considering demand response programs and energy storage system
}

\author{
Saeed ABAPOUR ${ }^{1}$, Sayyad NOJAVAN ${ }^{1}{ }^{\mathbb{D}}$, Mehdi ABAPOUR ${ }^{1}$
}

\begin{abstract}
This paper proposes a multi-objective benefit function for operation of active distribution systems considering demand response program (DRP) and energy storage system (ESS). In the active distribution system, active network management (ANM) is applied so that the distribution system equipment is controlled in real-time status based on the real-time measurements of system parameters (voltages and currents). The multi-objective optimization problem is solved using $\varepsilon$-constraint method, and a fuzzy satisfying approach has been employed to select the best compromise solution. Two different objective functions are considered as follows: benefit maximization of distribution company (DisCo); benefit maximization of distributed generation owner (DGO). To increase the benefits and efficient implementation of distributed generation (DG), DGO has installed battery as energy storage system (ESS) in parallel with DG unit. Consequently, DGO decides for the battery charging/discharging. DisCo has the ability to exchange energy with the upstream network and DGO. Also, DisCo focuses to study the effect of demand response program (DRP) on
\end{abstract}

CrossCheck date: 2 May 2017

Received: 2 July 2016/Accepted: 2 May 2017/Published online: 11 October 2017

(C) The Author(s) 2017. This article is an open access publication

Sayyad NOJAVAN

sayyad.nojavan@tabrizu.ac.ir

Saeed ABAPOUR

sa.abapour@gmail.com

Mehdi ABAPOUR

abapour@tabrizu.ac.ir

1 Faculty of Electrical and Computer Engineering, University of Tabriz, Tabriz, Iran total benefit function and consequently its influence on the load profile has been discussed. This model is successfully applied to a 33-bus radial distribution network.

Keywords Multi-objective benefit function, Active management, Distributed generation units, Demand response programs, Energy storage system

\section{Introduction}

From the operation cost point of view, supplying reliable electricity to the customers and improving the efficiency of power system and optimal scheduling of distributed generation (DG) units is important for both DG owner (DGO) and distribution company (DisCo). In an open access environment, the decisions related to DG investment/operation are taken by DG owners/operators and DisCo is responsible for maintaining the efficiency of the network. Hence, a win-win strategy is needed which not only promotes the DG benefit for DGO but also does not burden additional costs to DisCo.

Some of the existing works in the literature has investigated the technical and economic aspects of DG units. These aspects are as follows: active loss reduction [1], reducing the cost of curtailed energy [2], voltage profile improvement [3], enhancing the voltage stability [4], reducing the construction period [5] and reducing the cost of energy purchased from power market [6].

In most cases, distribution networks are expanded in a radial form. Therefore, the integration of DG units into distribution network may change the power flow in distribution feeders. For instance, unidirectional power flows will change to bidirectional power flows when the penetration level of DG becomes higher [7]. To achieve an 
appropriate status, DG units require a proper control and management system, as well as a proper operation model. This paper proposes active network management (ANM) of distribution network. Active management is an efficient method to reinforce distribution networks for connection of DG units and their operation [8]. Applying the ANM can help to reduce the losses, modify the peak load, control the voltage profile, decrease the DG curtailment, and postpone the need to reinforce the distribution network [9].

It should be noted that applying ANM is not sufficient for full load conditions and load growth. One of the proposed solutions in this paper is utilization of demand response program (DRP). DRP is defined as the changes of customer loads from their nominal value in response to incentive payments of operator [10]. Besides the financial benefits of DRP for customers (incentive payments), DRP can be utilized for enhancing power system stability. In [11], authors have investigated the effects of time-of-use rate of demand response programs on bidding strategy of electricity retailers. For this purpose, DRP has been developed on the load model. From the supply point of view, flexibility might bring significant improvements to the generation dispatch. From the demand point of view, flexibility could allow customers to benefit from reducing their energy bills. Consequently, in [12], authors compare how different modeling tools consider fixed and flexible loads in the dispatch optimization, and analyze their different strategies.

Besides DRP, this paper also studies the effects of energy storage system (ESS) on both DGO and DisCo benefits. In order to increase the benefits and efficient utilization of DG, DGO has installed an ESS such as battery in parallel with DG unit.

The advantages of ESS have been investigated in some works. In [13], the state of art battery energy storage technology and the methods of assessing their economic viability and their impact on power system operation have been discussed. In [14], the effect of ESS on stochastic energy procurement problem for large electricity consumers has been investigated when the defined cost function decreases. In [15], the ability of ESS to increase the amount of wind energy accepted onto a network is assessed and an analysis is conducted to determine the cost of produced energy through the ESS for a number of scenarios. The results show that ESS is able to increase the energy accepted onto a distribution network.

Although many of former works have analyzed the DG operation problem but a few of them have focused on the interaction between conflicting or convergent objectives of DGO and DisCo. Hence, in this paper, two objective functions are considered namely DisCo benefit maximization and DGO benefit maximization.
This paper proposes two solutions to improve the operation of distribution system. These solutions are DRP of consumers implemented by DisCo and active management of system. The proposed method provides a new model for short-term scheduling of distribution network in the presence of DG units. Both passive and active network managements have been simulated and the obtained results have been compared with together. The optimal DG operation problem is modeled as a mixed integer non-linear program which can be solved using commercial optimization packages like GAMS software.

The main contributions of this paper are four-fold:

1) Multi-objective function which considers the benefits of DisCo and DG owners and provide a win-win strategy for both parties.

2) Considering active management of system, demand response of costumers and battery charging/discharging decisions for proper status operation.

3) Time-variant load modeling based on time-of-use rate of demand response programs.

4) Utilizing of $\varepsilon$-constraint technique and fuzzy satisfying method to solve and choose the best collusion solution of multi-objective problem.

The rest of this paper is organized as follows:

In section 2, DRP based time-variant load model, multiobjective function for the DisCo benefit and DGO and related constraints are explained. In section 3 , concept of active network management and available implementation programs for optimal operation is expressed. In section 4, $\varepsilon$-constraint method and fuzzy decision maker are described. In Section 5, the proposed model is applied on the 33-bus radial distribution system and the simulation results are given and discussed. Finally, Section 6 summarizes the findings of this work.

\section{Mathematical formulation}

The used model in this paper considers costumer participation in DRP and installed ESS by DGO. The model is based on the optimal power flow and maximizes benefits of both DisCo and DGO at daily short-term period. Meanwhile, the model is simulated based on the daily load curve. The problem formulation is provided in four sub-sections as follows.

\subsection{Load and electricity price modeling}

The daily load variation is modeled by multiplication of two parameters. The first one is the base load $\left(P_{i, b a s e}^{D}, Q_{i, b a s e}^{D}\right)$. Each hour of a day is defined as a demand 
level. Therefore, there will be 24 hours or 24 demand levels which will be shown by $N_{h}$.

The second parameter is the demand level factor $\left(D L F_{h}\right)$. This factor defines the forecasted value of "load to peak load ratio" in each demand level and varies from 0 to 1. A typical $D L F_{h}$ is shown in Fig. 1. Therefore, the demand of the $i^{\text {th }}$ bus at the $h^{\text {th }}$ demand level is calculated as [16]:

$P_{i, h}^{D}=P_{i, \text { base }}^{D} \cdot D L F_{h}$

$Q_{i, h}^{D}=Q_{i, \text { base }}^{D} \cdot D L F_{h}$

$S_{i, h}^{D}=P_{i, h}^{D}+\mathrm{j} Q_{i, h}^{D}$

where $P_{i, h}^{D}, Q_{i, h}^{D}$ and $S_{i, h}^{D}$ are active, reactive and apparent load in bus $i$, and demand level $h$, respectively.

The price of purchased energy from main grid is determined by the market operation. This value changes during each demand level. In this paper, it is assumed that the price of electricity at the $h^{\text {th }}$ demand level can be determined as [16]:

$\lambda_{h}=\rho_{\text {base }} \cdot P L F_{h}$

where $\lambda_{h}$ is expected market price in level $h$ for active power and $\rho_{\text {base }}$ is the base price. A typical $P L F_{h}$ curve is illustrated in Fig. 1.

\subsection{Modeling time-of-use (TOU) rate of demand response program}

DisCo implements demand response programs to shift their consumers load from costly periods to inexpensive periods to decrease their costs. In this paper, it is assumed that the costumers participate only in TOU program and have a limited capability of shifting their demand. For example, their maximum capability

$y$ may be assumed to be 15 percent of total demand $\left(D R_{\max }=15 \%\right)$. DRP can be modeled as shown in Fig. 2 [11].

The dashed part of load in Fig. 2 is the section that does not participate in DRP and the other section is able to be

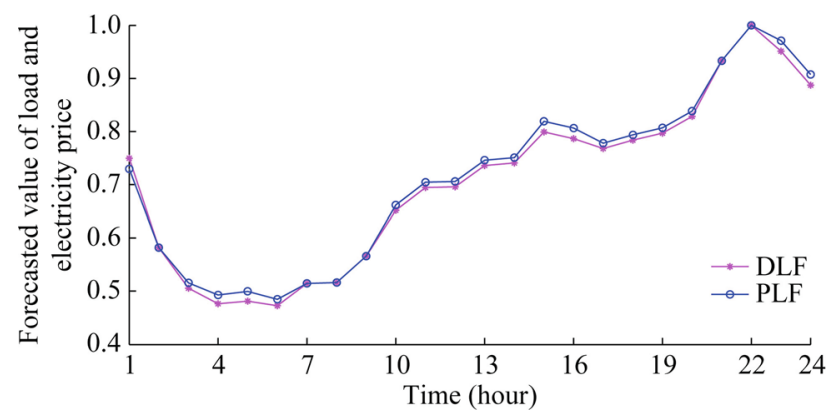

Fig. 1 Typical demand and price level factors

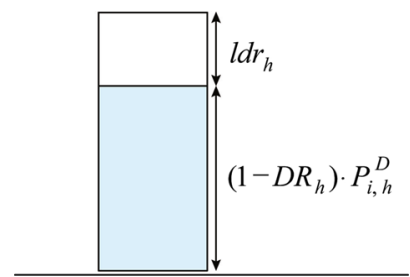

Fig. 2 Demand response based load modeling

shifted from one period to others due to price differences. Figure 2 can be defined mathematically as follows:

$P_{i, h}^{D}=\left(1-D R_{h}\right) \cdot P_{i, h}^{D}+l d r_{h}$

$P_{i, h}^{D}-P_{i, h}^{D R}=l d r_{h}=D R_{h} \cdot P_{i, h}^{D}$

where $l d r_{h}$ is the load value shifted by DRP at level $h$ and $D R_{h}$ factor shows the costumers participation in DRP. $P_{i, h}^{D R}$ is the load demand after DRP implementation.

\subsection{Objective function}

Operation model is a combination of DG units and other energy supply resources. DisCo has the ability to exchange energy with the upstream network and DGO. The DisCo's objective function in short-term operations framework is maximizing its energy benefits for one day. Equation (7) provides the mathematical formulation of a DisCo:

$$
\begin{aligned}
O F_{1}= & \sum_{t=1}^{N_{h}}\left\{\sum_{i=1}^{N_{\text {load }}} \rho_{\text {sell }}^{P} \cdot P_{i, h}^{D}+\sum_{i=1}^{N_{\text {load }}} \rho_{\text {sell }}^{Q} \cdot Q_{i, h}^{D}-\sum_{s s=1}^{N_{S S}} \lambda_{h} \cdot P_{h}^{s s}\right. \\
& -\sum_{s s=1}^{N_{S S}} \lambda_{Q f i x} \cdot Q_{h}^{s s}-\lambda_{h} \cdot P_{\text {loss }}^{\text {tot }}-\sum_{n=1}^{N_{D G}} \rho_{\text {sell }}^{P D O} \cdot P_{n, h}^{D G} \\
& \left.-\sum_{n=1}^{N_{D G}} \rho_{\text {sell }}^{Q D G O} \cdot Q_{n, h}^{D G}\right\}
\end{aligned}
$$

where $\rho_{\text {sell }}^{P}, \rho_{\text {sell }}^{Q}$ are price of the sold active and reactive power to the customers by DisCo, respectively; $P_{h}^{s s}, Q_{h}^{s s}$ are active and reactive power imported from grid in demand level $h$, respectively; $P_{n, h}^{D G}, Q_{n, h}^{D G}$ are active and reactive power injected by DG in bus $i$, demand level $h$; $P_{\text {loss }}^{\text {tot }}$ is total active power loss; $\rho_{\text {sell }}^{P D O}, \rho_{\text {sell }}^{Q D G O}$ are the price of sold active and reactive power by DGO to DisCo.

The first and second term of (7) are revenues from the energy sold to the customers by DisCo. The third term of (7) is the cost of power purchased from external network or energy market by DisCo, and its value depends on the electricity market price $\left(\lambda_{h}\right)$. The fourth term of (7) is the amount of payment for the reactive power purchased from the external network, and it is described as a pre-determined and fixed price $\left(\lambda_{Q f i x}\right)$. The fifth term describes the 
benefits or costs due to the variation of network power losses, which are affected by the power generation by DG units. Sixth and seventh terms show the amount of active and reactive energy purchased from DGO by DisCo, respectively. Also, DisCo has the ability to sell energy in the market. This condition occurs when $P_{h}^{s s}$ is negative.

The benefits of DGO come from selling energy to the DisCo. DGO has also invested on batteries. The price of sold energy by DGOs depends on the role they play in the market. They can have bilateral contracts with DisCo at fixed price or they can sell their output power at market price. In this paper, it is assumed that DGOs sells their generated power at fixed price. The benefit function of DGO is computed as:

$$
\begin{aligned}
O F_{2}= & \sum_{t=1}^{N_{h}}\left\{\sum_{n=1}^{N_{D G}} \rho_{s e l l}^{P D G O} \cdot P_{n, h}^{D G}+\sum_{j=1}^{N_{D G}} \rho_{\text {sell }}^{Q D G O} \cdot Q_{n, h}^{D G}+\sum_{k=1}^{N_{k}} \rho_{\text {sell }}^{P D G O} \cdot P_{k, h}^{d i s c}\right. \\
& -\sum_{k=1}^{N_{k}} \lambda_{h} \cdot P_{k, h}^{c}-\sum_{n=1}^{N_{D G}}\left(A_{n} P_{n, h}^{2}+B_{n} P_{n, h}^{D G}+C_{n}\right) \\
& \left.-\sum_{n=1}^{N_{D G}} Q_{n, h}^{D G} \cdot C T_{n}^{Q}-C_{k}^{d e g}\left(\sum_{k=1}^{N_{k}} \frac{P_{k, h}^{d i s c}}{\eta_{k}^{d i s c}}+\eta_{k}^{C} \cdot P_{k, h}^{c}\right)\right\}
\end{aligned}
$$

where $P_{k, h}^{c}, P_{k, h}^{\text {disc }}$ are the charge and discharge power of battery $k$ in demand level $h$; $C T_{n}^{Q}$ is reactive power cost of DG unit $n ; C_{k}^{d e g}$ is the degradation price of battery $k$; $\eta_{k}^{C}, \eta_{k}^{\text {disc }}$ is the charge and discharge efficiency of battery $k$; $A_{n}, B_{n}, C_{n}$ are the operation factors of DG units.

The first and second term of (8) are revenues from the energy sold to DisCo by DGO. The third term of (8) is revenues from the charged energy of batteries. The fourth term of (8) is the cost due to batteries charging. The fifth term represents the operating cost of DG unit's active powers. Here, a known structure of DG units for active power is considered. The last term captures the degradation cost of batteries at the $h^{\text {th }}$ hours for the $k^{\text {th }}$ battery due to the charging/discharging activities [13].

\subsection{Constraints and optimal power flow equations}

The power flow equations should be satisfied in the $i^{\text {th }}$ bus and at the $h^{\text {th }}$ hour as follows:

$$
\begin{gathered}
P_{h}^{s s}+P_{i, h}^{D G}-\left(\left(1-D R_{h}\right) \cdot P_{i, h}^{D}+l d r_{h}\right)+\sum_{k=1}^{N_{k}}\left(P_{k, h}^{d i s c}-P_{k, h}^{c}\right) \\
=V_{i, h} \sum_{j} V_{j, h}\left(G_{i j} \cos \delta_{i, h}+B_{i j} \sin \delta_{j, h}\right) \\
Q_{h}^{s s}+Q_{i, h}^{D G}-Q_{i, h}^{D}=V_{i, h} \sum_{j} V_{j, h}\left(G_{i j} \cos \delta_{i, h}-B_{i j} \sin \delta_{j, h}\right)
\end{gathered}
$$

where $V_{i, h}, \delta_{i, h}$ are the voltage magnitude and the voltage angle in bus $i$ and demand level $h$, respectively; $G_{i j}$ and $B_{i j}$ are conductance and susceptance between bus $i$ and $j$, respectively.

The voltage of each bus at the $h^{\text {th }}$ hour should be kept within the safe operating limits:

$V_{i}^{\min } \leq V_{i, h} \leq V_{i}^{\max }$

where $V_{i}^{\min }, V_{i}^{\max }$ are minimum and maximum voltage limitation of bus $i$, respectively.

The active and reactive powers of the substation are limited as follows [17]:

$P_{s s}^{\min } \leq P_{h}^{s s} \leq P_{s s}^{\max }$

$Q_{s s}^{\min } \leq Q_{h}^{s s} \leq Q_{s s}^{\max }$

where $P_{s s}^{\min }, P_{s s}^{\max }$ are minimum and maximum active power limitation of substation, respectively.

The DG units should be operated with considering the limits of their maximum installed capacity [18]:

$P_{D G}^{\min } \leq P_{i, h}^{D G} \leq P_{D G}^{\max }$

$Q_{D G}^{\min } \leq Q_{i, h}^{D G} \leq Q_{D G}^{\max }$

where $P_{D G}^{\min }, P_{D G}^{\max }$ are minimum and maximum active power of DG, respectively.

The power factor of DG units for all demand levels is assumed to be constant $[16,18]$ :

$\cos \phi=\frac{P_{i, h}^{D G}}{\sqrt{\left(P_{i, h}^{D G}\right)^{2}+\left(Q_{i, h}^{D G}\right)}}=$ const

The power flow through the line connected to nodes $i$, $j$ is limited by its thermal limit for all demand levels:

$S_{i j, h} \leq S_{i j}^{\max }$

where $S_{i j}^{\max }$ is maximum thermal limitation of branch between $i$ and $j$.

The tap setting of tap-changer $l$ at the $h^{\text {th }}$ demand level is limited by (18) as follows:

$T_{l}^{\min } \leq T_{l, h} \leq T_{l}^{\max }$

where $T_{l}^{\min }, T_{l}^{\max }$ are minimum and maximum setting of tap-changer $l$.

Equations (19)-(23) express DRP constraints. The moveable demand has a variable size in each period which is defined by $D R_{h}$ and it is represented by (19). $D R_{h}$ factor shows the customer participation in DRP.

$\sum_{h=1}^{N_{h}} l d r_{h}=\sum_{h=1}^{N_{h}} D R_{h} \cdot P_{i, h}^{D}$

Constraint (20) limits the demand increase in each time period.

$P_{i, h}^{D(i n c)} \leq \operatorname{inc}_{h} \times P_{i, h}^{D}$

Constraint (21) implies that during participation in DRP, 
the peak load (say occurs at hour 22) is greater than other load levels.

$$
\left(1-D R_{h}\right) \cdot P_{i, h}^{D}+l d r_{h} \leq\left(1-D R_{\left({ }^{\prime} 22^{\prime}\right)}\right) \cdot P_{i,\left(^{\prime} 22^{\prime}\right)}^{D}+l d r_{\left({ }^{\prime} 22^{\prime}\right)}
$$

Constraints (22) and (23) limit the maximum amount of $D R_{h}$ and $i n c_{h}$ in each period by " $D R_{\max }$ " and "inc $c_{\max }$ " which are considered to be $15 \%$.

$D R_{h} \leq D R_{\max }$

inc $_{h} \leq i n c_{\max }$

where $i n c_{\max }$ is maximum amount of demand increase.

Equations (24)-(27) express the ESS constraints [19]. Equation (24) shows the limits of charge and discharge power of battery $k$ in demand level $h$ so that if binary variable $b_{k, h}^{c}$ is 1 , it is in charging mode. Also, if binary variable $b_{k, h}^{\text {disc }}$ is 1 , it is in discharging mode. Otherwise it is in neither charging nor discharging mode. Equation (25) shows the capacity of battery $k$ in each demand level $h$ which is limited to a minimum and maximum value. Equation (26) indicates that the battery cannot be in charging and discharging mode at the same time. Equation (27) shows the changes of battery capacity at any demand level (hour) $h+1$ compared to demand level (hour) $h$.

$\left\{\begin{array}{l}0 \leq P_{k, h}^{c} \leq b_{k, h}^{c} P_{k, h}^{c, \max } \\ 0 \leq P_{k, h}^{\text {disc }} \leq b_{k, h}^{\text {disc }} P_{k, h}^{\text {disc,max }}\end{array}\right.$

$E_{k}^{\min } \leq E_{k, h} \leq E_{k}^{\max }$

$\left\{\begin{array}{l}b_{k, h}^{c}+b_{k, h}^{d i s c} \leq 1 \\ b_{k, h}, b_{k, h}^{d i c} \in\{1,0\}\end{array}\right.$

$\left\{b_{k, h}^{c}, b_{k, h}^{\text {disc }} \in\{1,0\}\right.$

$E_{k, h+1}=E_{k, h}+\left(\eta_{k}^{C} \cdot P_{k, h}^{c}-\frac{P_{k, h}^{d i s c}}{\eta_{k}^{d i s c}}\right)$

where $E_{k}^{\max }, E_{k}^{\min }$ maximum and minimum amount of operation capacity of battery $k$.

The objective is maximization of both $O F_{1}$ and $O F_{2}$ with considering constraints (9)-(27).

$$
\max \left\{\begin{array}{cc}
O F_{1} & O F_{2} \\
P_{h}^{s s}, Q_{h}^{s s}, P_{n, h}^{D G}, Q_{n, h}^{D G} & P_{n, h}^{D G}, Q_{n, h}^{D G}, P_{k, h}^{c}, P_{k, h}^{d i s c}
\end{array}\right\}
$$

$$
\text { (9) to (27) }
$$

\section{Active network management}

The hourly adjustment of transformer tap-changer and reactive power compensators in the distribution network can help getting smart of network, reduce the voltage deviation and power losses, and reduce the operation cost. Such a network is called active network. To record loads data in active network, automatic meter reading (AMR) devices are installed in loads location. AMR provides energy consumption data and sends them to the control center. Other data received by the control center are the proposed hourly price of energy by DG investors and predicted energy market price.

Fig. 3 shows the control and management of the distribution system based on ANM method. In active distribution system, it is assumed that a control center is located in the primary substation. According to Fig. 3, the state estimation process is carried out in the distribution system which receives the load data through the local and remote measurements.

At this stage, (1) DisCo regulates proper setting on-load tab changer (in secondary substation) and installed reactive power compensators so that all of the mentioned constraints are satisfied. (2) DisCo determines the value of bought (or sold) energy from (to) the energy market and also DG owner so that all of the mentioned constraints are satisfied.

Recently, many studies have been done in the field of active network management and have reviewed its advantages $[8,9,20]$.

Three ANM strategies can be applied which are described as follows [3]:

\section{1) Active power regulation of DG}

Active power regulation of each DG unit is one of the applied strategies to limit the voltage rise in distribution networks. In the conditions like high power generation and low load, the voltage increase problem may be solved by reduction of power generation of some DGs. Nevertheless, this strategy is rarely used because of the additional opportunity costs for DG units.

2) Active management of on-load-tap changer (OLTC)

The active management of the OLTC focuses on keeping the voltage profiles in the permitted range. Due to the continuous load variation that may cause voltage drop in the network, DG's generated power may create a voltage profile which increases at the DG connected node $[3,18]$.

\section{3) Using reactive power compensators (RPCs)}

By using RPC management, the reactive power flow in distribution system can be controlled and the voltage profile can be corrected when the safety voltage range exceeds. Also, RPC reduces the active power losses of network. The best control strategy is based on the application of coordinated and synchronized voltage and reactive power control. 


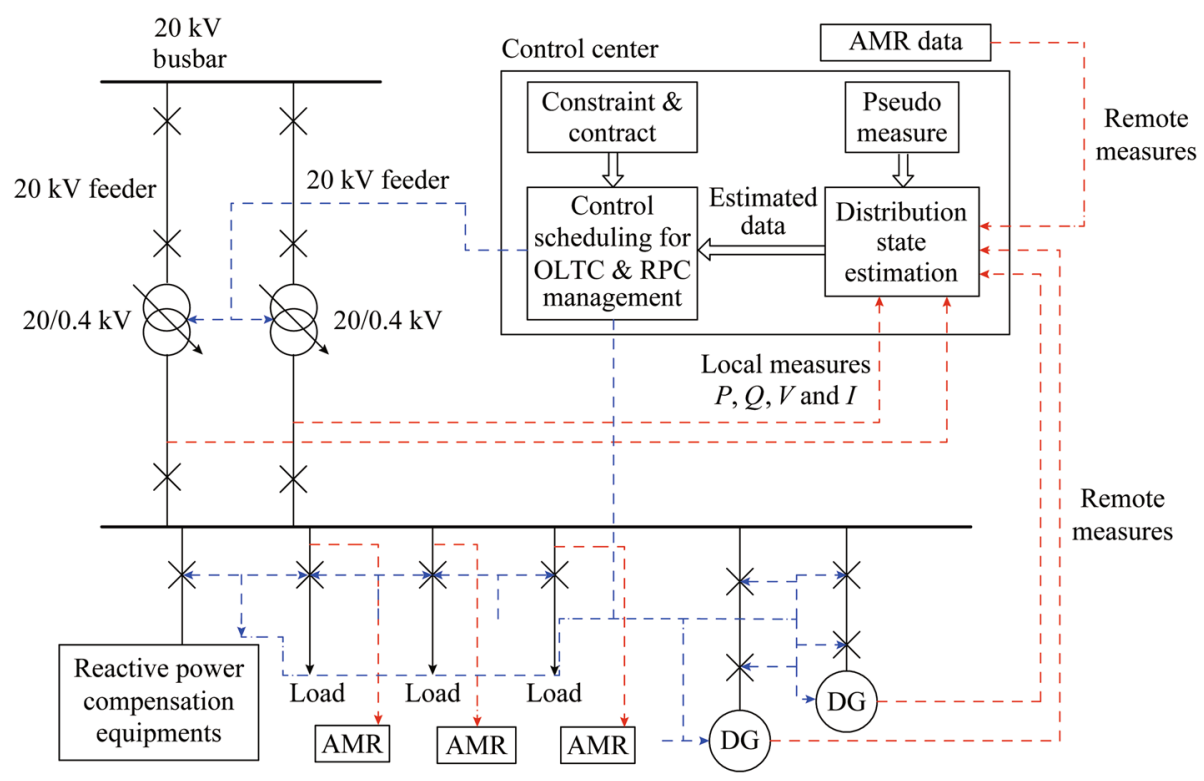

Fig. 3 Schematic of ANM in distribution system

In this paper, three operation modes of distribution systems are considered and compared with each other. These modes are described as follows:

\section{i) Mode 1 Conventional distribution networks}

The power flow is unidirectional from medium voltage (MV) substation to customers in low voltage levels. In this mode, no DG is connected to the network.

ii) Mode 2 Passive distribution networks

Both DG and RPC have been installed in the network to improve the technical characteristics. When the penetration level of DG becomes higher, the unidirectional power flows will probably change to bidirectional power flows. In this mode, ANM programs are not applied and the outputs of DG units and RPCs are not controlled by DisCo.

iii) Mode 3 Active distribution networks

Measurement devices are located at load buses and important nodes. Moreover, OLTC in MV substation is controlled automatically with the aim of measuring primary system parameters. ANM programs will be implemented for optimal and smart operation of distribution system.

\section{Problem solution method}

\section{$4.1 \varepsilon$-constraint method}

Various methods are used to solve multi-objective optimization problems. The proposed multi-objective model is solved using the $\varepsilon$-constraint method, which is an efficient technique to solve problems with a non-convex Pareto front. This method generates single objective subproblems by transforming all objectives except one of them into constraints. The lower bounds of these constraints are given by the epsilon-vector. The Pareto front can be obtained by varying the epsilon-vector. Therefore, in the proposed problem, of $f_{1}$ is optimized while $o f_{2}$ is considered as a constraint as follows [21]:

$$
\begin{aligned}
& O F=\max \left(o f_{1}\right) \\
& \left\{\begin{array}{l}
o f_{2} \geq \varepsilon \\
(9)-(27)
\end{array}\right.
\end{aligned}
$$

where $\varepsilon$ is a specified value between $O F_{2}^{\max }$ and $O F_{2}^{\min }$.

Fig. 4 and (28) show that $o f_{2}$ is constrained by the parameter $\varepsilon$. This parameter varies from the minimum value to the maximum value of $o f_{1}$ (i.e. from $O F_{2}^{\min }$ to $O F_{2}^{\max }$ ) gradually. For any value of $\varepsilon$, the modified single objective optimization problem (i.e. (28)) is solved, and the optimal solutions like point $C$ in Fig. 4 are obtained. It is noteworthy that in (28) the constraints of original multiobjective optimization problem, i.e. (9) - (27), are also included.

\subsection{Fuzzy satisfying method}

Fuzzy satisfying (or max (min)) method is a popular technique to select the best solution among the obtained $N_{p}$ Pareto optimal solutions. Suppose we have a problem with $N$ objectives to be minimized. The linear membership function for the $s^{\text {th }}$ solution of the $w^{\text {th }}$ objective function is defined as [22]: 


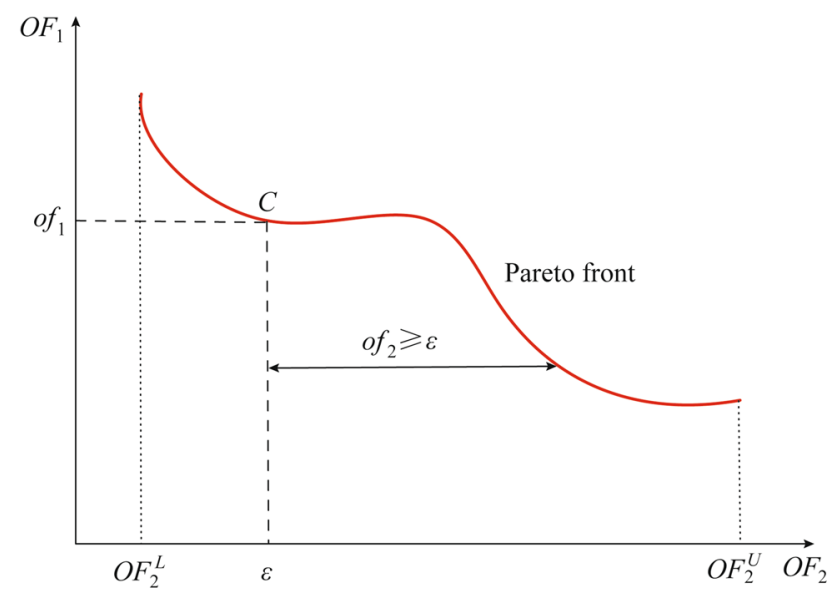

Fig. 4 Description of $\varepsilon$-constraint method

$\mu_{w}^{s}= \begin{cases}1 & O f_{w}^{s} \leq f_{w}^{\min } \\ \frac{O F_{w}^{\max }-o f_{w}^{s}}{O F_{w}^{\max }-O F_{w}^{\min }} & O F_{w}^{\min } \leq o f_{w}^{s} \leq O F_{w}^{\max } \\ 0 & o f_{w}^{s} \geq O F_{w}^{\max }\end{cases}$

where $O F_{k}^{\max }, O F_{k}^{\min }$ are the maximum and minimum values of the objective function $w$ in the solutions of Pareto optimal set; $\mu_{w}^{s}$ shows the optimality degree of the $s^{\text {th }}$ solution of the $w^{\text {th }}$ objective function. The membership function of the $s^{\text {th }}$ solution can be calculated using the following equation:

$\mu^{s}=\min \left(\mu_{1}^{s}, \mu_{2}^{s}, \ldots, \mu_{N}^{s}\right) \quad s=1,2, \ldots, N_{P}$

The solution with the maximum weakest membership function is the best solution. The corresponding membership function of this solution $\left(\mu^{\max }\right)$, is calculated as:

$\mu^{\max }=\max \left(\mu^{1}, \mu^{2}, \ldots, \mu^{N p}\right)$

With this background, the steps of solution method are briefly presented as follows:

1) Maximize $O F_{1}$ subject to all of equal \& unequal constraints. Calculate $\mathrm{OF}_{2}$ value which is in minimum value.

2) Maximize $\mathrm{OF}_{2}$ subject to all of equal \& unequal constraints. Calculate $O F_{1}$ value which is in minimum value. In this step, the minimum and maximum values of $O F_{1}$ and $O F_{2}$ are determined.

3) In the third step, one of the objectives $\left(O F_{2}\right)$ is defined as constraint for other objective as:

$\max o f_{1}^{s}$

s.t.

$\left\{\begin{array}{l}o f_{2}^{s} \geq \varepsilon+o f_{2}^{s-1} \\ \text { all of equal \& unequal constraints }\end{array}\right.$
4) Regarding the number of considered solutions (or iterations), objective function is changed in discrete steps between its minimum and maximum value. $\varepsilon$ is calculated as follow $\varepsilon=\frac{o f_{1}^{\max }-o f_{1}^{\min }}{\text { number of iteration }}$.

In the first iteration, $O F_{1}$ is at its maximum value and $O F_{2}$ is at its minimum value.

5) With increasing $O F_{2}$ for different iteration, $O F_{1}$ decreases. In this step, values of $O F_{1}$ and $O F_{2}$ for different iterations are calculated and a set of Pareto front is provided.

6) Fuzzy satisfying method is used to select the best iteration. The objectives are converted to per unit values in different iterations by (29). $\mu_{w}^{s}$ shows the optimality degree of the $s^{\text {th }}$ iteration of the $w^{\text {th }}$ objective function.

7) The membership function of the $s^{\text {th }}$ iteration can be calculated using the following:

$$
\mu^{s}=\min \left(\mu_{1}^{s}, \mu_{2}^{s}, \ldots, \mu_{N}^{s}\right) \quad s=1,2, \ldots, N_{P}
$$

8) The solution with the maximum weakest membership function is the best solution.

$$
\mu^{\max }=\max \left(\mu^{1}, \mu^{2}, \ldots, \mu^{N p}\right)
$$

\section{Assumptions and simulation results}

\subsection{Case study and problem assumptions}

In this study, simulations have been carried out on a 33-bus system edited by Baran and $\mathrm{Wu}$ [3]. The single-line diagram of this test system is represented in Fig. 5. The hypothetical voltage level of the substation is $12.66 \mathrm{kV}$ and the hypothetical capacity of the feeders is $8 \mathrm{MVA}$. The peak load is $4460 \mathrm{~kW}$ and $2760 \mathrm{kvar}$.

In this problem, DG investment was carried out by private sector. $\mathrm{DG}_{1}$ and $\mathrm{DG}_{2}$ are installed at the node 11 and 33, respectively. The DG units have a capacity of 1.5 MW. RPC1 and RPC2 are installed at the buses 17 and 33, respectively. $\mathrm{RPC} 1$ is $0.8 \mathrm{Mvar}$ and $\mathrm{RPC} 2$ is $1.7 \mathrm{Mvar}$ to reactive power compensation. Two energy storage system (ESS) (for example the battery\#1 and the battery\#2) are

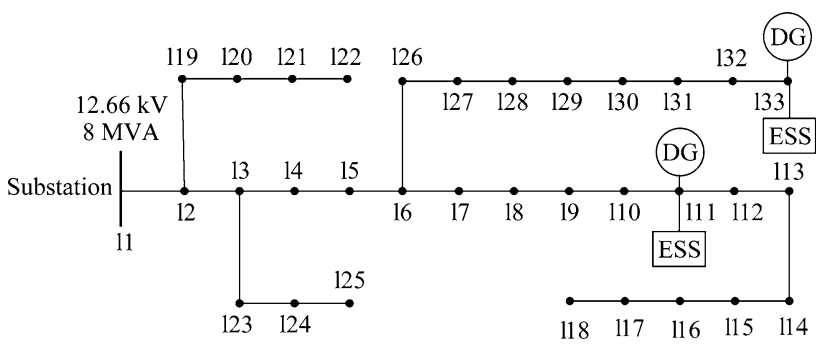

Fig. 5 Single line diagram of 33-bus system 
installed in parallel with the DG units. For this purpose, two battery units with the capacity of $0.5 \mathrm{MWh}$ are considered with charging/discharging power rating of $200 \mathrm{~kW}$. The minimum and maximum energy stored in the battery are $100 \mathrm{kWh}$ and $450 \mathrm{kWh}$, respectively. Operating period is equal to 24 hours. The base price of energy purchased from the grid is assumed to be $72 \$ / \mathrm{MWh}$. The forecasted values of each load level $D L F_{h}^{f}$, are shown in Fig.1. Duration of each load level is one hour. The remainder of parameters is listed in Tables 1, 2 and 3.

\subsection{Simulation results}

To illustrate the DRP effect on the proposed model, two case studies have been evaluated. In Case A, the short-term scheduling model is studied without considering DRP. In Case $\mathrm{B}$, the same problem is solved considering the DRP to analyze its effects on the load profile and total benefit function.

\subsubsection{Case A: short-term scheduling without DRP}

The Pareto front is obtained for 33-bus test system without considering demand response. Table 4 summarizes the obtained Pareto solutions for the case. To compare and

Table 1 DG units cost data [23]

\begin{tabular}{lllll}
\hline Bus with DG & $A\left(\$ / \mathrm{MW}^{2}\right)$ & $B(\$ / \mathrm{MW})$ & $C(\$)$ & $C T_{j}^{Q}(\$ / \mathrm{Mvar})$ \\
\hline 11 & 0.0075 & 36 & 28.5 & 4.28 \\
33 & 0.0075 & 40 & 22 & 8.5 \\
\hline
\end{tabular}

Table 2 Economical and technical data

\begin{tabular}{llll}
\hline Parameters & Values & Parameters & Values \\
\hline$V_{i}^{\max }($ p.u. $)$ & 1.05 & $\rho_{\text {sell }}^{P}(\$ / \mathrm{MWh})$ & 85 \\
$V_{i}^{\min }$ (p.u. $)$ & 0.95 & $\rho_{\text {sell }}^{Q}(\$ / \mathrm{Mvarh})$ & 52 \\
$\mathrm{PF}_{\mathrm{DG}}$ & 0.95 & $\rho_{\text {sell }}^{\text {DGO }}(\$ / \mathrm{MWh})$ & 67 \\
$\rho_{\text {base }}$ & 72 & $\lambda_{Q f i x}(\$ / \mathrm{Mvarh})$ & 42 \\
\hline
\end{tabular}

Table 3 Battery unit parameter

\begin{tabular}{llll}
\hline Parameters & Values & Parameters & Values \\
\hline$E_{k}^{c a p}(\mathrm{MWh})$ & 0.5 & $P_{k, h}^{c, \max }(\mathrm{MW})$ & 0.2 \\
$E_{k}^{\min }(\mathrm{MWh})$ & 0.1 & $P_{k, h}^{d i s c, \max }(\mathrm{MW})$ & 0.2 \\
$E_{k}^{\max }(\mathrm{MWh})$ & 0.45 & $\eta_{k}^{C}$ & 0.95 \\
$C_{k}^{d e g}(\$ / \mathrm{MWh})$ & 2.7 & $\eta_{k}^{\text {disc }}$ & 0.85 \\
\hline
\end{tabular}

demonstrate the effectiveness of active management method in the short-term scheduling of distribution system, these results for ANM method are also calculated and listed in Table 4. It is assumed that the location of the installed DGs in both PM and ANM methods are identical. By using min-max fuzzy satisfying method, it is found from Table 4 (The bold values is the Pareto optimal solution)that the best compromise solution is Solution 13, with the maximum weakest membership function of 0.6. The corresponding DisCo and DGO benefits are equal to 4225.10 and 1763.26 dollar per day in passive management, respectively. In active management, these values will be 6022.21 and 1703.56 dollar per day for DisCo and DGO benefits, respectively. It should be also noted that the Solution 1 corresponds to the DisCo benefit maximization case, i.e. in the Solution, only DisCo benefit is maximized, and the maximum value of DisCo benefit is 6452.40 dollar per day in the active network.

Table 5 shows the optimal setting of OLTC in the substation and RPCs in the proposed model for the 33-bus distribution network. Equipment's setting is shown for any assumed load level during one day. The aim of this work is to create a suitable condition for operation of a distribution network along with DG units. Settings will be according to the constraints of voltage and reactive power RPCs.

Fig. 6 indicates the received power value from upstream grid and generated power by DG units. The received active power value decreases with considering DG power generation. The received active power from the upstream network will be reduced during the hours 10 to 21 . DisCo provides electrical energy in the cheap hours of the day from upstream network and during the hours of $10 \mathrm{am}$ to 24 pm, DisCo will deal with the DG owner. Fig. 6 (b) indicates the output power of DG units for daily scheduling period in the passive network. DGO is only able to sell energy to DisCo. According to Fig. 7 (b), in the hours that the DGO's offer is less than the energy market's one, DisCo will venture into buying from DGO. DisCo can also sell the energy purchased from DGO at the energy market, if that is profitable. Fig. 6 (c) illustrates the purchased power from the upstream network by DisCo in active network. The purchased reactive power by DisCo is zero due to the installed RPCs in the network. In other words, DisCo provides reactive power itself by RPCs.

Fig. 6 (d) shows the generated power of DG units in active network. The sold reactive power by DGO will be zero. Therefore, DGO benefit is lower compared to passive network.

Fig. 7 (a) shows the voltage profiles for the traditional, passive and active system. The voltage curve is shown for the peak load which occurs at the hour twenty-two. According to Fig. 7 (a), the voltage drop increases as the distance from substations increases in the traditional 
Table 4 Pareto optimal solutions for short-term scheduling of active distribution system without DRP (Case-A). The bold values is the Pareto optimal solution

\begin{tabular}{|c|c|c|c|c|c|c|c|c|c|c|}
\hline \multirow[t]{2}{*}{ No. } & \multicolumn{5}{|c|}{ Passive network management } & \multicolumn{5}{|c|}{ Active network management } \\
\hline & $\begin{array}{l}\text { DisCo profit } \\
\text { (\$/day) }\end{array}$ & $\begin{array}{l}\text { DGO profit } \\
\text { (\$/day) }\end{array}$ & $\mu_{1}$ (p.u.) & $\mu_{2}$ (p.u.) & $\min \left(\mu_{1}, \mu_{2}\right)$ & $\begin{array}{l}\text { DisCo profit } \\
\text { (\$/day) }\end{array}$ & $\begin{array}{l}\text { DGO profit } \\
\text { (\$/day) }\end{array}$ & $\mu_{1}$ (p.u.) & $\mu_{2}$ (p.u.) & $\min \left(\mu_{1}, \mu_{2}\right)$ \\
\hline 1 & 4667.09 & 680.1131 & 1 & 0.101 & 0.101 & 6452.405 & 390.7996 & 0.98 & 0.016 & 0.016 \\
\hline 2 & 4667.09 & 680.1131 & 1 & 0.101 & 0.101 & 6450.816 & 466.2984 & 0.98 & 0.05 & 0.05 \\
\hline 3 & 4667.09 & 680.1131 & 1 & 0.101 & 0.101 & 6438.791 & 578.7772 & 0.97 & 0.10 & 0.10 \\
\hline 4 & 4664.361 & 785.8268 & 0.997 & 0.15 & 0.15 & 6423.644 & 691.2561 & 0.96 & 0.15 & 0.15 \\
\hline 5 & 4636.522 & 894.4311 & 0.976 & 0.20 & 0.20 & 6400.543 & 803.735 & 0.94 & 0.20 & 0.20 \\
\hline 6 & 4606.537 & 1003.035 & 0.952 & 0.25 & 0.25 & 6374.527 & 916.2139 & 0.92 & 0.25 & 0.25 \\
\hline 7 & 4568.776 & 1111.64 & 0.922 & 0.30 & 0.30 & 6343.784 & 1028.693 & 0.90 & 0.30 & 0.30 \\
\hline 8 & 4526.411 & 1220.244 & 0.889 & 0.35 & 0.35 & 6300.46 & 1141.172 & 0.86 & 0.35 & 0.35 \\
\hline 9 & 4478.005 & 1328.848 & 0.851 & 0.40 & 0.40 & 6255.505 & 1253.65 & 0.83 & 0.40 & 0.40 \\
\hline 10 & 4423.42 & 1437.452 & 0.809 & 0.45 & 0.45 & 6205.39 & 1366.129 & 0.79 & 0.45 & 0.45 \\
\hline 11 & 4366.131 & 1546.057 & 0.764 & 0.50 & 0.50 & 6149.862 & 1478.608 & 0.75 & 0.50 & 0.50 \\
\hline 12 & 4295.002 & 1654.661 & 0.708 & 0.55 & 0.55 & 6087.827 & 1591.087 & 0.70 & 0.55 & 0.55 \\
\hline 13 & 4225.106 & 1763.265 & 0.653 & 0.60 & 0.60 & 6022.214 & 1703.566 & 0.65 & 0.60 & 0.60 \\
\hline 20 & 3500.26 & 2523.495 & 0.085 & 0.95 & 0.085 & 5322.396 & 2490.918 & 0.10 & 0.95 & 0.10 \\
\hline
\end{tabular}

Table 5 Optimal settings of OLTC and RPCs in the proposed model for the ANM method without considering DRP

\begin{tabular}{|c|c|c|c|c|c|c|c|}
\hline Hour & $\mathrm{RPC}_{1}$ (Mvar) & $\mathrm{RPC}_{2}$ (Mvar) & OLTC (p.u) & Hour & $\mathrm{RPC}_{1}$ (Mvar) & $\mathrm{RPC}_{2}$ (Mvar) & OLTC (p.u) \\
\hline 1 & 0.575 & 1.228 & 0.99 & 13 & 0.553 & 1.207 & 0.99 \\
\hline 2 & 0.409 & 0.960 & 1.05 & 14 & 0.557 & 1.217 & 0.99 \\
\hline 3 & 0.361 & 0.845 & 1.05 & 15 & 0.599 & 1.321 & 0.98 \\
\hline 4 & 0.340 & 0.797 & 1.05 & 16 & 0.605 & 1.303 & 0.98 \\
\hline 5 & 0.342 & 0.781 & 1.04 & 17 & 0.568 & 1.262 & 0.99 \\
\hline 6 & 0.337 & 0.791 & 1.05 & 18 & 0.588 & 1.296 & 0.98 \\
\hline 7 & 0.363 & 0.852 & 1.05 & 19 & 0.597 & 1.317 & 0.98 \\
\hline 8 & 0.365 & 0.855 & 1.05 & 20 & 0.619 & 1.368 & 0.99 \\
\hline 9 & 0.397 & 0.930 & 1.05 & 21 & 0.692 & 1.539 & 0.99 \\
\hline 10 & 0.469 & 1.057 & 1.02 & 22 & 0.752 & 1.655 & 0.98 \\
\hline 11 & 0.515 & 1.129 & 1.00 & 23 & 0.705 & 1.573 & 0.98 \\
\hline 12 & 0.516 & 1.132 & 1.01 & 24 & 0.659 & 1.464 & 0.99 \\
\hline
\end{tabular}

networks. The proper integration of DG units in the network can improve the voltage profile.

In this paper, DGO is responsible for making decision to the batteries charge/discharge programs. Fig. 7 (b, c) shows the hourly battery power output in the passive and active network. These values are subject to the battery charger limit (i.e., $[-200 \mathrm{~kW},+200 \mathrm{~kW}]$ ) at any time step. In normal conditions, because the grid electricity price is cheaper in the early morning, the battery bank starts to store as much energy as possible. Accordingly, the battery banks start to return the stored energy to the distribution network at the peak load times. DGO must be commensurate with the need DisCo attempted to charge and discharge the batteries.

\subsubsection{Case B: short-term scheduling considering DRP}

In this case, short-term scheduling problem is solved considering DRP. It should be noted that the consumer load profile is changed. In this case, the corresponding DisCo and DGO benefits are equal to 4368.16 and 1768.31 dollar per day in passive management, respectively. In active 


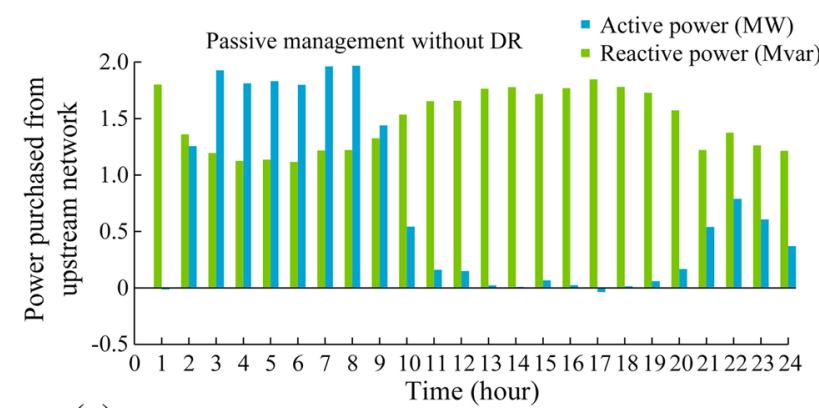

(a) Purchased power from the upstream network by DisCo in passive network

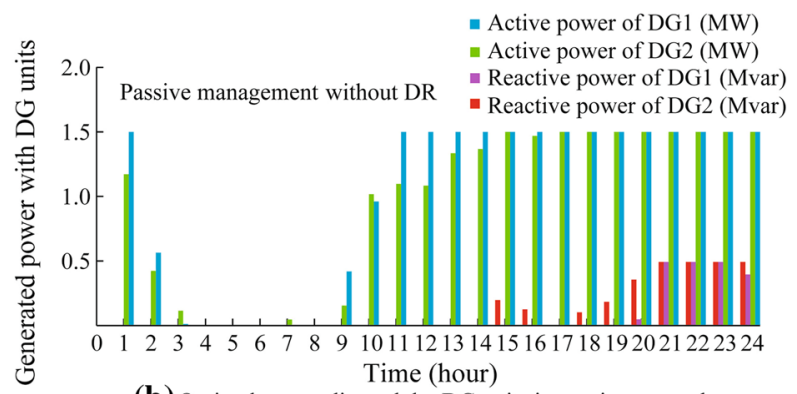

(b) Optimal power dispatch by DG units in passive network

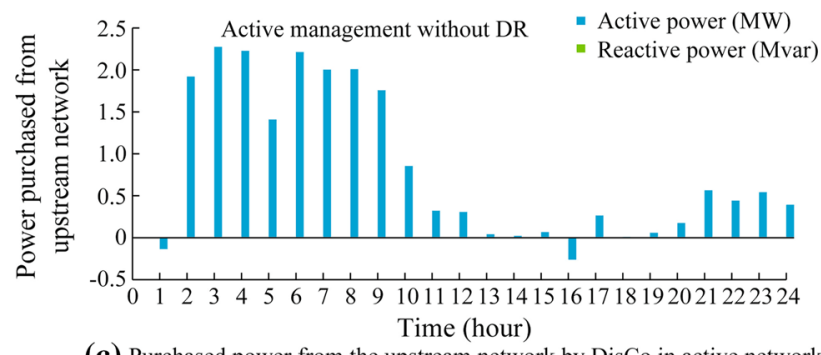

(c) Purchased power from the upstream network by DisCo in active network

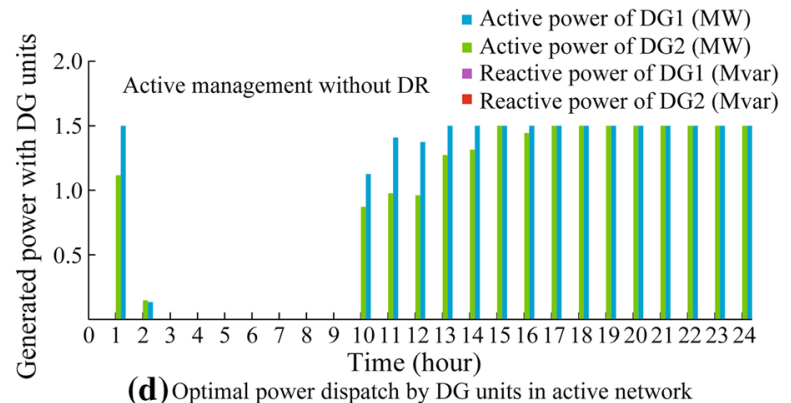

Fig. 6 Power exchange for passive and active network without DRP

management, for DisCo and DGO benefits these values will be 6176.78 and 1687.76 dollar per day, respectively. Results indicate that DisCo and DGO benefits have increased with considering DRP in passive network. In active network, because the sold reactive power by DGO will be zero, the result is slightly different so that DGO benefit is decreased.

Fig. 8 indicates the purchased (or sold) power values of DisCo from (to) the upstream grid and DGO considering DRP. Between the hours 10 to 21 , the purchased active

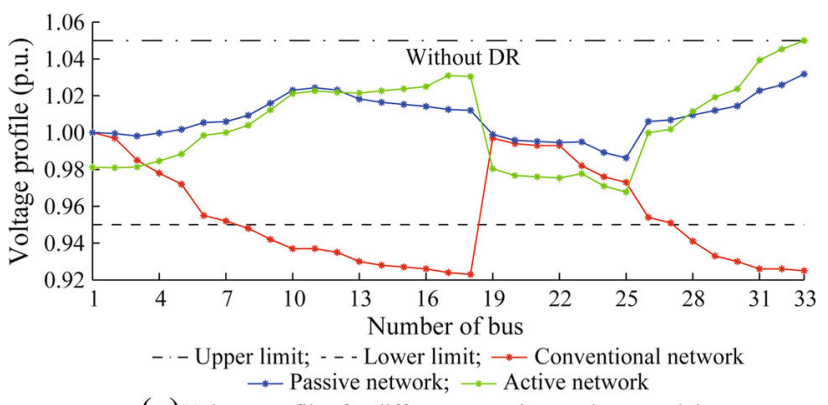

(a) Voltage profiles for different operation modes at peak hours

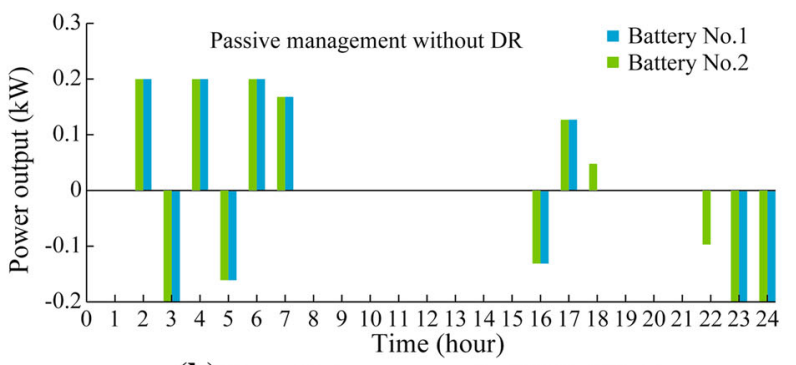

(b) Charge/discharge program by DGO in PM state

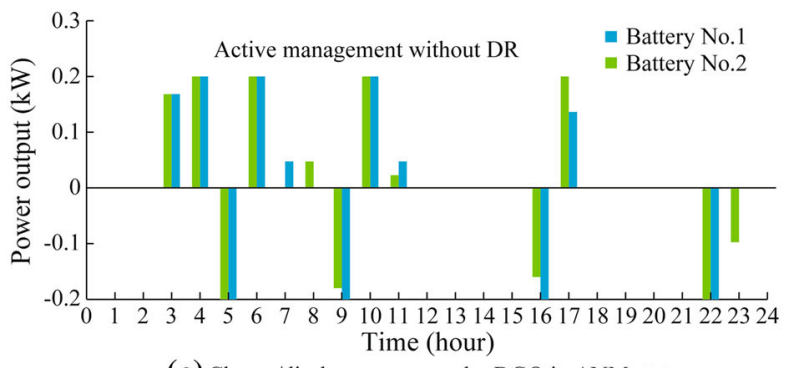

(c) Charge/discharge program by DGO in ANM state

Fig. 7 Results of voltage profiles and output power of batteries without DRP

power from the upstream network will be reduced. According to Fig. 8 (a, c), between the hours 15 to 20, DisCo sells the purchased energy from DGO, in the energy market. Hence, the transfer of power is reversed. DisCo provides electrical energy in the cheaper hours of the day from upstream network and DGO during the day and sells to customer in their region. Fig. 8 (b) indicates the output power of DG units for daily scheduling period in passive network. According to Fig. 8 (b), at the hours that the DGO's offer is less than the energy market's one, DisCo will venture into buying from DGO. Fig. 8 (c) indicates the purchased power from the upstream network by DisCo in active network. The purchased reactive power by DisCo is zero due to the installed RPCs in the network. In other words, DisCo provides reactive power itself by RPCs. Fig. 8 (d) shows the generated power of DG units in active network. The sold reactive power by DGO will be zero.

It should be noted that the consumer load profile is changed. Fig. 9 shows the load profile with and without considering DRP. 


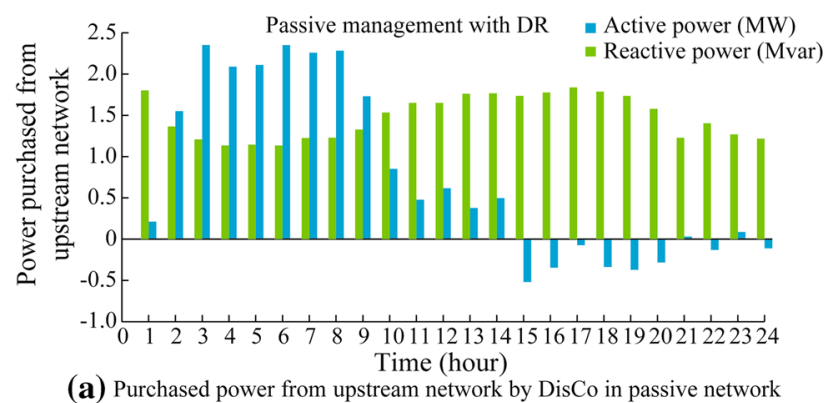

(a) Purchased power from upstream network by DisCo in passive network

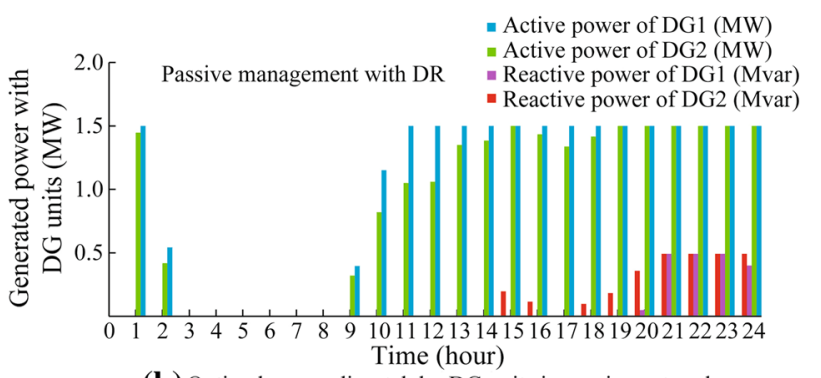

(b) Optimal power dispatch by DG units in passive network

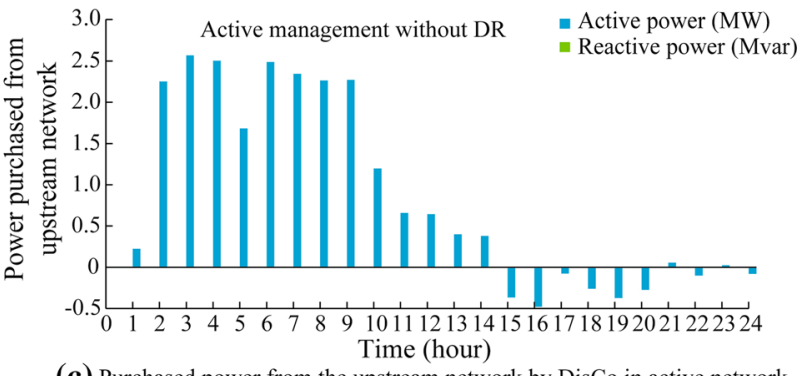

(c) Purchased power from the upstream network by DisCo in active network

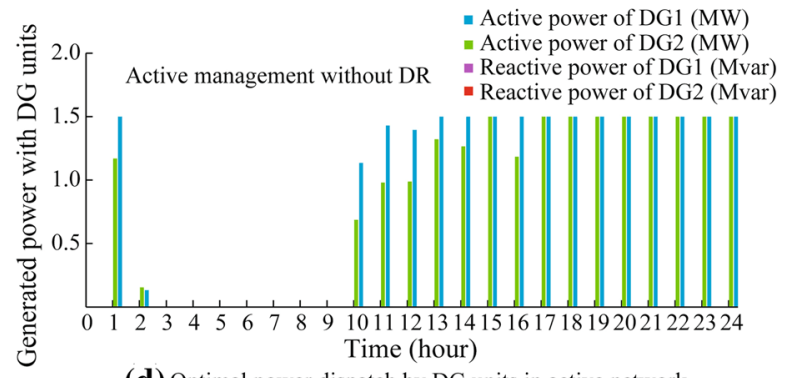

(d) Optimal power dispatch by DG units in active network

Fig. 8 Results for passive and active network considering DRP

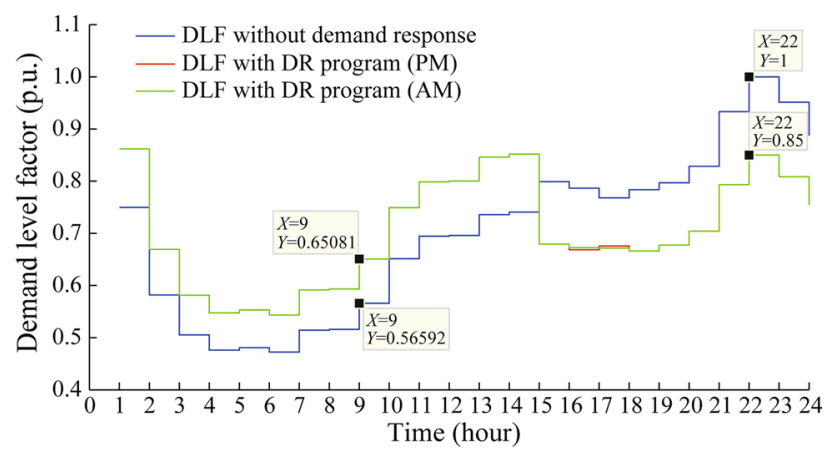

Fig. 9 Demand level factor with and without DRP in active network

\section{Conclusion}

In this paper, the active network management was presented for short-term scheduling of distribution system along with DG units. Benefits function of DisCo and DGO are optimized simultaneously in a multi-objective optimization framework. To validate the efficiency of proposed approach, two case studies were used to assess the effects of DRP in increasing the DisCo benefit function. It can be concluded from the obtained results that the voltage profile and network power losses are intensely dependent on the management method of system. One of the main conclusions of this work is that active management is more beneficent than passive management. Implementation of DRP in the system could also flat the load profile. The batteries are charged at the cheap hours by the network and the stored energy is sold by DGO in the peak and expensive hours to DisCo. The results show that in cheaper hours DGO cannot compete with the network market and DisCo will choose the best option for the energy purchase. However, the investment of private sector in the network will improve the voltage and load profile and will reduce the power loss.

Open Access This article is distributed under the terms of the Creative Commons Attribution 4.0 International License (http:// creativecommons.org/licenses/by/4.0/), which permits unrestricted use, distribution, and reproduction in any medium, provided you give appropriate credit to the original author(s) and the source, provide a link to the Creative Commons license, and indicate if changes were made.

\section{References}

[1] Abapour S, Zare K, Mohammadi-Ivatloo B (2014) Evaluation of technical risks in distribution network along with distributed generation based on active management. IET Gener Transm Distrib 8(4):609-618

[2] Zangeneh A, Jadid S, Rahimi-Kian A (2010) Normal boundary intersection and benefit-cost ratio for distributed generation planning. Eur Trans Electr Power 20(5):1430-1440

[3] Abapour S, Zare K, Mohammadi-Ivatloo B (2015) Dynamic planning of distributed generation units in active distribution network. IET Gener Transm Distrib 9(12):1455-1463

[4] Esmaili M, Chaktan FE, Shayanfar HA (2014) Optimal placement of distributed generations considering voltage stability and power losses with observing voltage-related constraints. Appl Energy 113:1252-1260

[5] Pouresmaeil E, Montesinos-Miracle D, Gomis-Bellmunt O et al (2010) A multi-objective control strategy for grid connection of $\mathrm{dg}$ (distributed generation) resources. Energy 35(12):5022-5030

[6] Soroudi A, Ehsan M (2010) Multi-objective planning model for integration of distributed generations in deregulated power systems. Iran J Sci Technol Trans B Eng 34(3):307-324

[7] Lund P (2012) Large-scale urban renewable electricity schemes-integration and interfacing aspects. Energy Convers Manag 63:162-172 
[8] Gill S, Kockar I, Ault GW (2014) Dynamic optimal power flow for active distribution networks. IEEE Trans Power Syst 29(1):121-131

[9] Zhang J, Cheng H, Wang C (2008) Technical and economic impacts of active management on distribution network. Int $\mathbf{J}$ Electr Power Energy Syst 31(1):130-138

[10] Mazidi M, Zakariazadeh A, Jadid S et al (2014) Integrated scheduling of renewable generation and demand response programs in a microgrid. Energy Convers Manag 86:1118-1127

[11] Nojavan S, Mohammadi-Ivatloo B, Zare K (2015) Optimal bidding strategy of electricity retailers using robust optimization approach considering time-of-use rate demand response programs under market price uncertainties. IET Gener Transm Distrib 9(4):328-338

[12] Neves D, Pina A, Silva CA (2015) Demand response modeling: a comparison between tools. Appl Energy 146:288-297

[13] Luo X, Wang J, Dooner M et al (2015) Overview of current development in electrical energy storage technologies and the application potential in power system operation. Appl Energy 137:511-536

[14] Nojavan S, Aalami HA (2015) Stochastic energy procurement of large electricity consumer considering photovoltaic, wind-turbine, micro-turbines, energy storage system in the presence of demand response program. Energy Convers Manag 103:1008-1018

[15] Wu K, Huan Z, Sicheng A et al (2015) Optimal coordinate operation control for wind-photovoltaic-battery storage powergeneration units. Energy Convers Manag 90:466-475

[16] Soroudi A, Ehsan M (2011) A possibilistic-probabilistic tool for evaluating the impact of stochastic renewable and controllable power generation on energy losses in distribution networks-a case study. Renew Sustain Energy Rev 15(1):794-800

[17] López-Lezama J, Contreras J, Padilha-Feltrin A (2012) Location and contract pricing of distributed generation using a genetic algorithm. Int J Electr Power Energy Syst 36(1):117-126

[18] Abapour S, Zare K, Mohammadi-Ivatloo B (2013) Maximizing penetration level of distributed generations in active distribution networks. In: IEEE smart grid conference (SGC), pp 113-118
[19] Nguyen DT, Bao LL (2014) Optimal bidding strategy for microgrids considering renewable energy and building thermal dynamics. IEEE Trans Smart Grid 5(4):1608-1620

[20] Calderaro V, Conio G, Galdi V et al (2014) Active management of renewable energy sources for maximizing power production. Int J Electr Power Energy Syst 57:64-72

[21] Mohseni-Bonab SM, Rabiee A, Mohammadi-Ivatloo B (2016) Voltage stability constrained multi-objective optimal reactive power dispatch under load and wind power uncertainties: A stochastic approach. Renew Energy 85:598-609

[22] Soroudi A, Mohammadi-Ivatloo B, Rabiee A (2014) Energy hub management with intermittent wind power. Large scale renewable power generation. Springer, Berlin, pp 413-438

[23] Algarni AS, Bhattacharya K (2009) Disco operation considering DG units and their goodness factors. IEEE Trans Power Syst 24(4):1831-1840

Saeed ABAPOUR received the B.Sc. and M.Sc. degrees in electrical engineering from the University of Zanjan, Zanjan and the University of Tabriz, Tabriz, Iran, in 2011 and 2013, respectively. He is currently a Ph.D. student at the University of Tabriz. His research interests include active distribution networks operation, energy management, multi- microgrids, and electrical vehicles.

Sayyad NOJAVAN received the B.Sc., M.Sc. and Ph.D. degrees in electrical power engineering from University of Tabriz, Tabriz, Iran, in 2010, 2012 and 2017, respectively. His research areas include smart grid, energy management, energy storage system, power system operation, renewable energy sources.

Mehdi ABAPOUR is an Assistant Professor with the School of Electrical and Computer Engineering, University of Tabriz. His research interests include reliability and power system planning. 